\title{
"Au-dessus des nuages, la liberté doit être infinie...»
}

\section{Christoph Bosshard}

Dr med., membre du Comité central de la FMH, responsable du département Données, démographie et qualité / Académie suisse pour la qualité en médecine (ASQM)

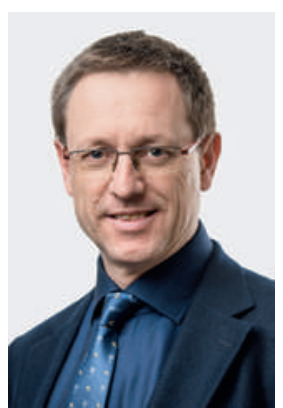

Jouir d'une liberté infinie, la sonder, l'explorer, s'en délecter, vivre ses rêves: voilà ce que chante Reinhard Mey dans sa chanson Über den Wolken (Au-dessus des nuages). Mais tous ceux qui ont un lien concret avec l'aviation savent combien chaque vol est réglementé et chaque étape décortiquée en fonction de la sécurité. La sécurité des passagers est la priorité numéro un, au même titre que la sécurité des patients en médecine. Il n'est donc pas surprenant que de nombreux éléments qui contribuent à la sécurité en aviation se retrouvent aussi en médecine. Le CIRS (Critical Incident Reporting System) en est un exemple éloquent, tout comme le projet Safe Surgery Saves Lives de Sécurité des

Nous, médecins, n'avons aucune chance de réussite si nous ne mettons pas le patient, et toutes ses spécificités, au centre.

patients Suisse qui aide à réduire les erreurs grâce à des processus et des listes de contrôle contraignants et clairement structurés. Le corps médical se prête d'ailleurs au jeu par un engagement intense: le nombre d'hôpitaux souhaitant participer à ce dernier projet a été nettement supérieur à ce que les ressources allouées permettaient d'assumer.

Certains doivent désormais penser que reprendre les réglementations en matière d'aéronautique ouvre la voie à une maîtrise parfaite de toutes nos exigences en matière de qualité. Les organisateurs d'un grand nombre d'ateliers sur la qualité en médecine s'offrent d'ailleurs volontiers les services d'experts en aéronautique. Mais plus ces échanges sont approfondis, plus il apparaît clairement que les standardisations et les parallèles entre la médecine et l'aéronautique atteignent leurs limites. Un pilote est formé sur un type d'appareil disposant d'un cockpit standard. Rien que là, les médecins font face à une différence de taille: tous leurs patients sont des individus uniques. De plus, ceux qui nous préoccupent le plus, et impactent le plus notre système de solidarité, souffrent de maladies qui majoritairement sont en interaction avec leur environnement social. Nous ne pouvons pas, et nous ne voulons pas, ne traiter que le «diabète» ou que l'«arthrose». Nous, médecins, n'avons aucune chance de réussite si nous ne mettons pas le patient, et toutes ses spécificités, au centre de notre action.

Dès lors, une approche médicale axée sur le patient constitue tout autant un critère de qualité que la mise en œuvre systématique de processus standardisés, là où ils existent. L'un ne devant cependant pas exclure l'autre. Comme l'erreur humaine constitue le plus souvent (et aussi dans l'aviation) la cause principale d'accident, nous devons développer des instruments pour y répondre. Un pas dans cette direction a été accompli par exemple avec l'implémentation de la procédure "IQM de revue par des pairs», mise actuellement en place par $\mathrm{H}+$ avec le soutien de la FMH et de l'ASDSI (Association Suisse des Directrices et Directeurs des Services Infirmiers).

C'est aussi dans ce sens que la FMH soutient Sécurité des patients Suisse et la semaine d'action du 14 au 18 septembre 2015, à laquelle elle participe par différentes actions, dont notamment ce numéro spécial du Bulletin des médecins suisses entièrement consacré à cette question. Les articles présentés vous en apprendront davantage sur les activités concrètes des diffé-

En matière de sécurité des patients, les standardisations et les parallèles entre médecine et aéronautique ont leurs limites.

rentes organisations impliquées. Vous trouverez également des informations complémentaires sur les sites www.securitedespatients.ch et www.asqm.ch. Je vous remercie de votre engagement quotidien en faveur de ces aspects de la sécurité des patients. 